\title{
CLASSIFICATION OF NEPHRITIS.
}

\section{By JOHN GRAY, M.D. \\ (Reader in Pathology, British Post-Graduate Medical School.)}

In some ways, classification of nephritis is a rather discouraging, as well as a difficult, task, for it is likely that with fuller knowledge of causation we will eventually be enabled to improve on and to simplify any classification possible at present. In the meantime, it is very difficult to decide on the respective merits of the numerous classifications suggested. On the other hand, however, it is true that the multiplicity of the terms which have been used to define various groups of nephritic cases rather tends to exaggerate the number of really important differences of opinion. Many opposing terms in use imply little fundamental difference of opinion, and simply indicate a preference for some particular label for a given type of case. On many points there is, except for this question of nomenclature, fairly general agreement, and it is perhaps as well to begin by indicating some of these, before entering on any discussion of those of a more controversial nature.

Few, for instance, would object to the statement that the terms acute, subacute, and chronic nephritis provide a useful primary grouping for the majority of cases of true nephritis; nor, one thinks, would many take exception to the statement that, of the allied diseases which must be considered with nephritis, easily the most important is non-nephritic hypertension-whatever term is used to describe that condition. Differences of opinion concern chiefly the exact definition of these groups, and the number and nature of further groups which it is necessary to add.

\section{Acute Nephritis. (Diffuse). \\ Synonyms. Acute Toxic Nephritis (Russell). Acute Diffuse Glomerulo-nephritis.}

Of the term acute nephritis it can be said that, used without a further qualifying adjective, it implies to most clinicians and pathologists a well defined condition. It is characterized during life by a relatively short period-say, up to about four weeks, of evidence of progressive renal inflammation-albuminuria with casts and red cells, raised blood pressure and odema; and the kidneys show an acute, diffuse non-purulent inflammation. Glomeruli, tubules and interstitium all become affected, in variable ratio-but the glomerular changes-largely an "overcellularity" due to endothelial proliferation, sometimes with polymorphonuclear infiltration-are generally regarded as primary to the others*.

It is true that the ratio between the changes in the various renal elements varies widely in different cases; and that the exact changes in the glomeruli, tubules or interstitium may also vary sufficiently to merit fully some subdivision from a purely pathological point of view; but it seems doubtful if such sub-division is of material advantage clinically. This is all the more true if, as is possible, many of these observed histological variations are dependent more on the duration and severity of the disease than on any more fundamental differences $\left(\mathrm{Gray}^{(5)}\right)$. It does not seem, for instance, that the terms glomerular, tubular or glomerulotubular nephritis are much more than convenient abbreviations of the histological descriptions of individual cases.

* No attempt is made in this paper to give any further histological detail than that involved incidentally in the discussion of classification. 


\section{Acute Focal Nephritis.}

\section{Synonym. One variety of Symptomatic Nephritis (Russeli).}

There is, however, a well-recognized group of acute cases which are quite distinctly different from ordinary " acute nephritis," namely acute focal nephritis. This has been described under a variety of names, but without much real difference of opinion as to the nature of the condition, either clinically or pathologically. By some such term as this is denoted a condition of focal, primarily glomerular, inflammation due to trapping of circulating bacteria in certain of the glomerular capillaries, and characterized clinically by nothing more than a variable degree of hæmaturia. Such a condition is probably very common. It is often merely an incidental feature in a generalized infection, and is probably without important later sequelæ, though these cases have been suggested as providing a possible explanation of some of the more chronic renal lesions. From its very nature, it is a lesion which in minor degree must often escape attention at the time of the attack. It probably corresponds to a great extent to the acute hæmorrhagic nephritis of some observers (Hill ${ }^{(7)}$; Capon ${ }^{(3)}$, etc.).

\section{Focal Embolic Nephritis.}

Synonym. Embolic Non-suppurative Focal Nephritis (Löhlein).

In bacterial endocarditis, there occurs the well recognized focal embolic nephritis (Löhlein ${ }^{(8)}$ ), where some of the lesions are due to actual emboli of thrombus lodging in glomerular capillaries. Here, too, the condition is sufficiently clearly defined to be generally accepted as a separate entity. It has to be borne in mind, of course, that true diffuse nephritis may also complicate subacute bacterial endocarditis (Baehr \& Lande ${ }^{(1)}$, etc.).

\section{Subacute Nephritis.}

Synonyms. (a) Intense Toxic Nephritis with maximum reaction in an intermediate stage, and (b) Less intense and more chronic variety of intense Toxic Nephritis (Russell).

(b) Subacute Diffuse Glomerulo-nephritis (extra-capillary type), and (b) Subacute Diffuse Glomerulo-nephritis (intra-capillary type) (Volhard and Fahr).

The term subacute nephritis is perhaps less widely used than the preceding terms, and, when used, there is a greater variability in the type of case to which it is applied. If a subject of acute nephritis does not die in the attack, there are several alternative later possibilities. Recovery is probable, or a latent stage, followed by manifest chronic nephritis, may set in; or evident nephritis may continue. It is to cases where the last alternative occurs that the term subacute nephritis can conveniently be applied. In the sense that there is no sudden or clear-cut alteration in signs or symptoms, the use of the term may seem redundant, and some simply regard an acute nephritis which fails to clear up as having become a chronic nephritis. The term is thus perhaps not an essential one in classification, but it does serve to describe a distinctive clinical phase, and it can be identified histologically in the kidneys of such cases. Indeed, its onset is marked by the appearance in the kidney of new types of change, indicating irreparable renal damage of some degree, and so of great prognostic significance. 
Two grades of severity can be recognized:

(a) The first type comprises cases which show albuminuria, œdema and hypertension, with or without uræmia, and which have persisted for more than about a month without entering a phase where the albuminuria and cedema have become trifling or absent. Death, mainly from renal failure, generally occurs within a very few months. Histologically, the kidneys of such cases are distinguished by active proliferation of the epithelium of Bowman's capsule (" crescent" formation), some increase of interstitial connective tissue, and some hyperplasia of the epithelium of the convoluted tubules.

(b) Other cases, starting more insidiously and again showing marked albuminuria and œdema, with some hypertension, show a slower onset of renal failure, and may last up to about a year, before death occurs either from renal failure or, more frequently, from a combination of renal and cardiac failure. In these latter cases, the glomerular changes are usually "intra-capillary" (Volhard and Fahr), i.e., the epithelial proliferations of Bowman's capsule are absent, and the enlarged glomeruli show marked endothelial proliferation with closure of many loops. Lipoid degeneration in convoluted tubules is often a very marked feature.

\section{Nephrotic Nephritis.}

In certain cases, the evidence of renal insufficiency, and even hypertension, may be unusually delayed and slight in degree. Death from intercurrent infection may in such cases reveal kidneys where the glomerular changes are entirely intracapillary and relatively slight, while accompanying increase of interstitial tissue is also slight, and lipoid infiltration of tubules is great. Such cases are really similar in nature to those in the preceding group, and certainly are not the "genuine nephrosis" of Volhard and Fahr. They may conveniently be referred to as cases showing a phase of " nephrotic nephritis."

\section{Chronic Nephritis.}

This very wide group includes at one extreme cases which have progressed from a manifest acute nephritis which has never cleared up completely in a clinical sense. They have been subject throughout to recurrences of some degree of œedema, with a variable but persistent albuminuria and hæmaturia; death from uræmia being likely within about two years. At the other end of the scale are included cases where chronic nephritis may be manifest for some months or years before death, and where no history of acute nephritis can be elicited-the presumption being that a latent course of many years has preceded its first revelation. Edema other than cardiac is absent or inconspicuous throughout, and albuminuria is slight, hæmaturia microscopic only. Between these two extremes, there are all intermediate grades of case.

This wide variation in the clinical picture is paralleled by an equally great range in the renal changes. And, naturally, many attempts have been made to subdivide this very wide group.

A notable advance in this direction was made by Russell ${ }^{(9)}$ in I929, when, by distinguishing "acris" and "repens" types of chronic nephritis, she defined two main histological groups, one in which the changes were relatively diffuse, and another in which they were more focal, with a "reticular" distribution of the fibrosis. While it was not invariably possible to relate these two histological types to separate clinical pictures, cases of the diffuse "acris" group more frequently gave a history of a previous acute attack, and at all stages were more liable than 
the focal reticular (repens) ones to show œdema. These tendencies are borne out by the experience of most workers. The value of her further sub-division of these groups is probably mainly in purely histological classification.

It is not possible to be certain whether these diffuse and focal forms indicate a different ætiology. There is some temptation to regard the focal form as being a sequel to an attack or attacks of acute focal nephritis, but this does not seem likely, and the general concensus of opinion is that acute focal nephritis seldom leads to serious subsequent renal damage (Hadfield \& Garrod ${ }^{(6)}$ ). It seems more likely that a similar ætiological factor has in the two types of case progressed with a variable vigour. Where a very high percentage of the glomeruli suffer irreparable damage in the early stages, there should be more likelihood of clinical evidence of an "acute" attack, and more chance of the damage to the other units being too extensive to allow of as complete or prolonged subsidence of symptoms; and also too extensive damage to allow of much hyperplasia of the relatively less affected units. A relatively diffuse histological picture and an earlier death, would in these cases be in accordance with theoretical expectations. But where fewer glomeruli suffer irreparable initial damage from the inflammation, insidious onset and clinical latency would seem more likely, together with a capacity on the part of less damaged units show more hyperplasia and so produce histologically a focal picture; and, probably, survival sufficiently long to show a smaller total renal weight at death. But these considerations are merely speculative.

(a) Essential Hypertension, and (b) Malignant Hypertension.

Synonyms.

Essential Hypertension :- Primary Ischæmic Nephritis (Russell). Nephrosclerosis of Benign Hypertension (Volhard \&. Fahr). Arteriolosclerotic Kidney.

Malignant Hypertension :- Malignant Nephrosclerosis.

That numerous cases of hypertension are not of nephritic origin has now been long agreed. The term arteriolosclerotic kidney, among others, is applied to the kidneys of cases of this "essential" hypertension. In the majority of cases there is no difficulty, either clinically or histologically, in classification. Clinically, in such cases, the disease is generally found in older people than is chronic nephritis, and the hypertension is unaccompanied by renal insufficiency. Death occurs from heart failure or cerebral hæmorrhage. At autopsy, the kidneys may appear practically normal or may be more or less contracted (often, in the latter case, of the "red granular" type). They are very unlikely in any case to be as smail as the smaller of the kidneys of chronic nephritis. Histologically, arterial sclerosis, and, especially, arteriolosclerosis, dominate the picture. The interlobular and other arteries show a thick sclerotic intima and a hypertrophied or partly fibrosed media. The afferent arterioles of the glomeruli show marked hyaline-fatty thickening. Focally, especially under the capsule, there are numbers of hyaline glomeruli with some associated increase, or apparent increase, of interstitial tissue. But most renal units show very little damage. This is a picture in no way likely to be confused with any other condition. In the severity of the arteriolar change it is quite different from the unimportant " arteriosclerotic" or senile kidney, and, on the other hand, no changes suggestive of nephritis are present.

But there are two occasional sources of difficulty, namely, the distinction of certain of the cases from "malignant" hypertension, and of certain others from chronic nephritis. 
The difficulty in the first case arises partly from the variable meaning given to the term " malignant." Where the clinician uses the term to include all cases of essential hypertension which, during the terminal period of heart failure, and because of that heart failure, show a secondary renal insufficiency, it is difficult to associate that clinical division with a reliable histological contrast between the malignant and non-malignant types of case. While it is doubtful if any case of essential hypertension (non-malignant) shows renal insufficiency apart from heart failure - there is too much satisfactory renal tissue-the vascular involvement may sometimes be sufficiently extensive to mean that, when the heart does fail, an inadequate renal circulation may result, with, therefore, manifestations of renal insufficiency. All that can safely be said of such cases is that one expects in them to find the renal contraction, the vascular changes and the ischæmic fociall of them features possible in non-malignant hypertension - to be present in severe degree. Quite possibly, too, there may be some hypertrophy of the healthy units. But these differences of degree are not very satisfactory as a means of classification. In some, also, there may be mild endothelial proliferation, though this is a feature which would, according to many pathologists, indicate the presence of nephritis.

If, however, a much narrower definition is given to the term " malignant," it becomes easier for the pathologist to supply a parallel classification. On such a basis one would include as malignant only cases of non-nephritic hypertension with a severe and rapid clinical course,* marked hypertensive retinal changes, and renal insufficiency occurring more or less independently of heart failure. In these cases, rare in this country at least, histological examination of the kidneys may show glomerular necroses, and often arteriolar necroses with endarteritis of the rather larger arteries. The affected glomeruli may be entirely necrotic with karyorrhexis of the nuclei, or, more often, the necrosis may affect only the primary branches of the afferent arteriole, near the root of the glomerulus. Affected arterioles show a "smudgy" staining of the whole thickened wall with eosin (Fishberg ${ }^{(4)}$ ) or other stains and nuclei may show karyorrhexis or disappearance. Rather larger arteries frequently show a cellular proliferation of the intima (endarteritis).

Another rare type of histological picture may possibly be related to this one. Sternberg ${ }^{(10)}$ and others describe renal lesions which are practically those of polyarteritis nodosa, in cases with greatly hypertrophied hearts and high blood pressure. The writer encountered a case recently where this combination was present. In it, the polyarteritis involved the small arteries of practically every organ, and was clearly an inflammatory lesion. The heart was greatly hypertrophied. Clinically, the case had been one of essential rather than of malignant hypertension, and death occurred from cardiac failure. Such cases are so unusual, however, that it is difficult to reach any satisfactory conclusion as to their correct interpretation.

The second difficulty sometimes encountered in essential hypertension is the separation of occasional cases from chronic nephritis. The resemblance can only be to cases of chronic nephritis which have been of such slow progress that the signs of glomerulitis are slight and the amount of vascular change unusually great. Such nephritic cases may have shown marked renal insufficiency only when the heart was failing. Many (e.g. Russell) claim that the presence of any endothelial 
proliferation at all in the tufts is of itself sufficient evidence that the kidney in question was nephritic - either as an explanation of the whole picture or as a condition superimposed on essential hypertension. There are, however, occasional cases which, dying without renal insufficiency, show extreme renal arterial and arteriolar changes, and are typically arteriolosclerotic except that some glomeruli show a little endothelial proliferation, and some swelling of the capillary basement membranes. These one is inclined to admit as instances of essential hypertension.

\section{Lipoid Nephrosis.}

Lipoid nephrosis is generally defined clinically as a condition characterized by copious albuminuria, severe œdema, a chronic course, hypercholesterolæmia, normal blood urea and blood pressure. Most agree that this picture, when encountered, frequently represents merely a nephrotic phase in a case of nephritis. Probably a true lipoid nephrosis, unrelated to nephritis, does exist, but it seems that it must be a rare disease, and most cases which for a time mimic the picture turn out to be nephritic. The histological picture described in the condition is one of extensive lipoid deposit in the tubular epithelium. This change is generally held to be unassociated with appreciable glomerular inflammation or interstitial increase. Glomerular changes have, indeed, been described, but it is doubtful if the cases concerned were actually true lipoid nephrosis, $\left(B^{2} l^{(2)}\right)$.

RETERENCES.

(1) Baehr \& Lande (1920), J. Amer. Med. Ass., Lxx7, 789.

(2) Bell, E. T. (1929), Amer. J. Path., 7, 539 \& 587.

(3) Capon, N. B. (1926), Arch. Dis. Child, 1, 141

(4) Fishberg. A. M. (1934), Hypertension \& Nephritis. Baillière, Tindall \& Cox, London.

(5) Gray, J. (1933), Med. Research Coun., Spec. Rep. Ser., No. 178.

(6) Hadfield, G. \& Garrod, L. P. (1934), Recent Advances in Pathology. J. \& A. Churchill, Ltd., London.

(7) Hill. L. W. (1919), J. Amer. Med. Ass., 1xxili, 1747.

(8) Löhlein, M. (1910), Med. Klinik, vi, 370.

(9) Russell, D. S. (1929), Med. Research Coun., Spec. Rep. Ser., No. 142

(10) Sternberg, B. (1929), Arch. Int. Med., xliv, 272. 